\title{
Cesarean scar pregnancy: a case report
}

\begin{abstract}
A caesarean scar pregnancy (CSP), which is considered a type of ectopic pregnancy, occurs when the embryo is implanted in the myometrium at the site of a previous caesarean section (CS) scar
\end{abstract}

Volume 3 Issue 3 - 2015

\author{
Stoyan Karadakov, Antonopoulou K, \\ Jordanova $\mathrm{E}$ \\ Department of Obstetrics and Gynecology, General Hospital of \\ Imathia- Unit of Veria, Greece
}
Correspondence: Stoyan Karadakov, Ethnikis Antistaseos 5B avenue Thessaloniki-Greece, 55I34,,Tel 306997291834, Email:karadak@abv.bg

Received: July 22, 2015 | Published: December II, 2015

\section{Introduction}

A caesarean scar pregnancy (CSP), which is considered a type of ectopic pregnancy, occurs when the embryo is implanted in the myometrium at the site of a previous caesarean section (CS) scar. ${ }^{1}$ The exact incidence of CSP is unknown, as very few cases have been reported in the literature. ${ }^{2}$ The first case was described in 1978 by Larsen and Solomon and its incidence is increasing due to the rising rates of caesarean section and the higher accuracy of ultrasonography for diagnosis. ${ }^{3}$ Since 1978 until 2001, only 19 cases had been reported, by 2006 there were 155 cases and by 2011 the number of cases reported was 751 , showing a rapid increase in the incidence of this type of ectopic pregnancy. ${ }^{3}$ Jurkovic et al. $^{2}$ have estimated a prevalence of $1: 1800 .^{2}$ A case series estimated an incidence of 1:2226 of all pregnancies, with a rate of $0.15 \%$ in women with a previous CS and a rate of $6.1 \%$ of all ectopic pregnancies in women who had at least one caesarean delivery. ${ }^{1,2,4}$

\section{Case}

A 31 year old Gravida 3 Para 2 in her 10 th +0 week of gestation was brought to our $\mathrm{Ob} / \mathrm{Gyn}$ department by the ambulance because of acute onset lower abdominal pain followed by a syncopal episode. The patients' pregnancy was confirmed by an obstetrical ultrasound performed 2 weeks prior to her presentation in our department and was described as a singleton intrauterine pregnancy. Her past obstetrical history included 2 uncomplicated caesarian sections 4 and 1 years earlier. On presentation the patient was hypotensive and tachycardic (BP: 90/60, HR: 102). The patient was resuscitated with IV fluids. On speculum examination a normal vagina and a closed cervix were noted. On bimanual examination the size of the uterus was consistent with a 10 week pregnancy. There was diffuse tenderness on palpation of the abdomen with decreased bowel sounds and Kehr sign positivity. (Acute pain in the tip of the shoulder due to the presence of blood or other irritants in the peritoneal cavity when a person is lying). Her hematocrit and hemoglobin were $23.2 \%$ and $7.5 \mathrm{~g} / \mathrm{dL}$ respectively. On transabdominal ultrasound a round gestational sac with a live fetus (CRL 10w +1 ) were revealed (Figure 1) (Figure 2). The sac was located in the lower anterior uterine segment. Free fluid was noted in the cul-de-sac.

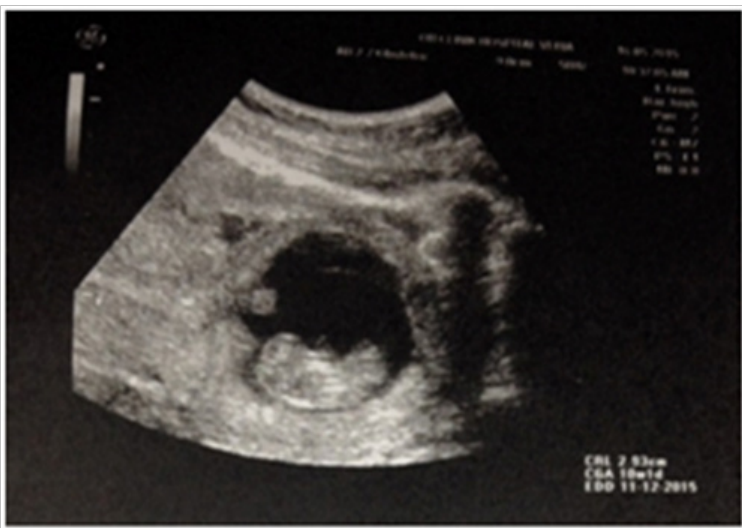

Figure I CRL: IOwId.

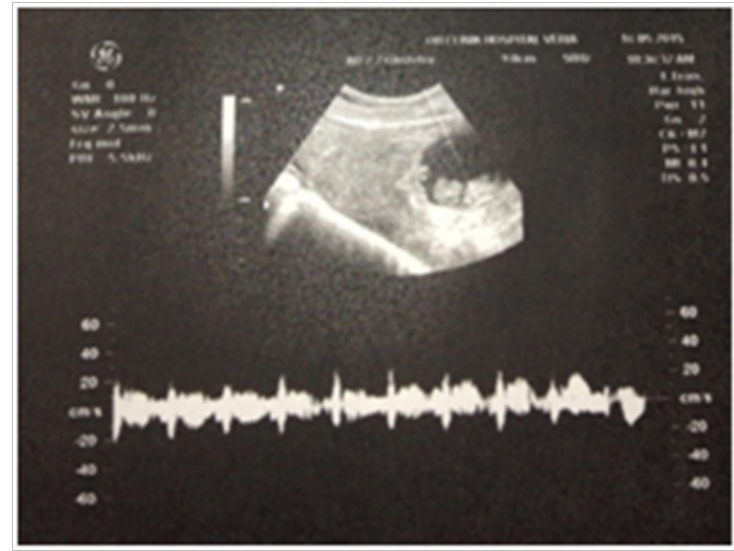

Figure 2 FHR.

Because of the equivocal findings of the ultrasound an abdominopelvic $\mathrm{CT}$ scan with contrast was performed which revealed a large amount of free intraperitoneal fluid and identified with certainty that the source of bleeding was the lower pelvis (Figure 3) (Figure 4).

Immediate laparotomy was performed. A ruptured uterus at the site of the previous caesarian section was found. The embryo inside the intact gestational sac was found floating in the abdominal cavity (Figure 5). Repair of the uterus was not possible; consequently a subtotal hysterectomy was performed. 


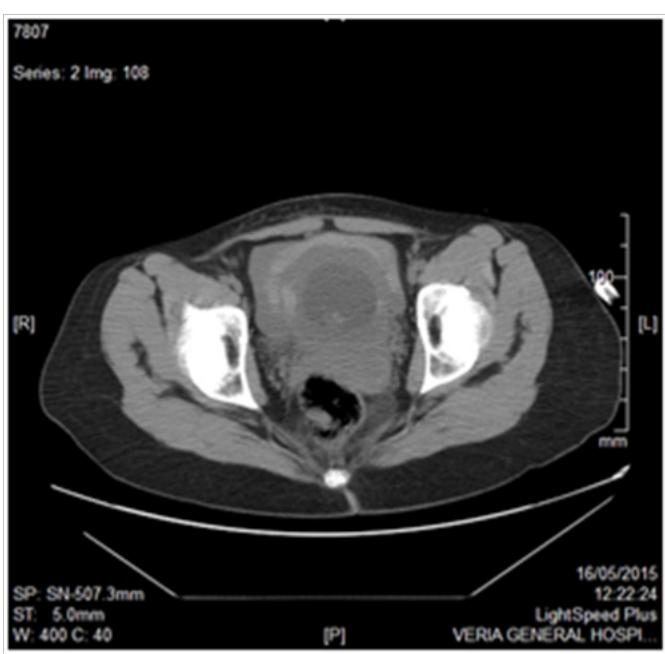

Figure 3 ICT scan without contrast-pelvic section.

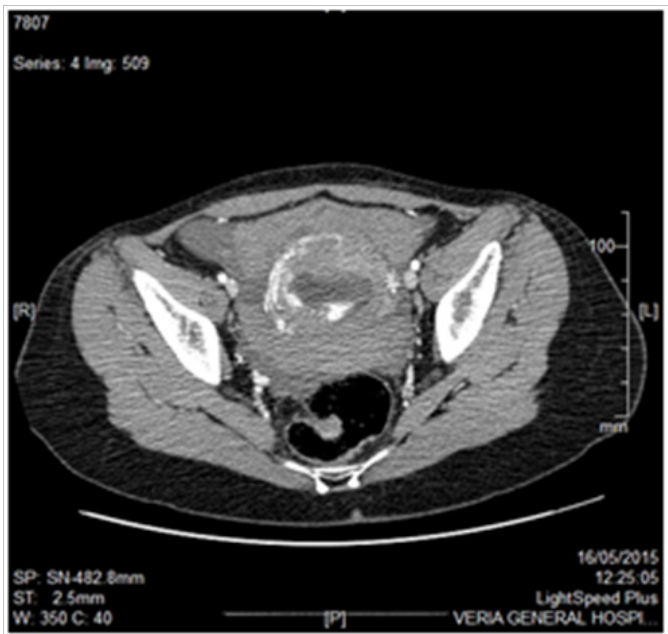

Figure 4 ICT scan with contrast-pelvic section.

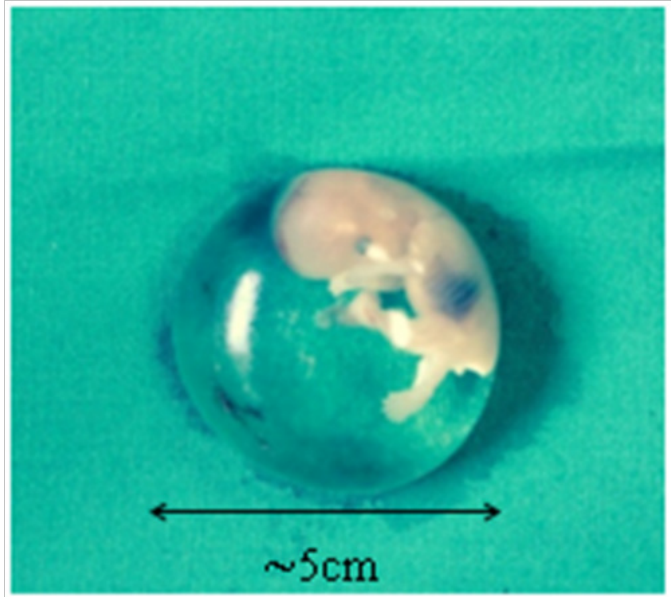

Figure 5 Embryo inside the intact gestational sac was found floating in the abdominal cavity.

\section{Discussion}

The implantation of the embryo in the scar of a previous caesarian section is the rarest form of ectopic pregnancies. ${ }^{3}$ In spite of this, because of the potential deleterious effects of a CSP on a patient, such as hemorrhage secondary to placental invasion and vascularity and rupture at the site of implantation, this possibility should always be considered in women who had a previous C-section, especially if the implantation of the embryo has occurred in the lower uterine segment.

Early diagnosis by transvaginal ultrasound is the gold standard. ${ }^{5}$ MRI can accurately detect the exact location of pregnancy, thus confirming the diagnosis. ${ }^{5}$ Although it is not difficult to obtain the proper diagnosis, it can easily be missed, if the examiners index of suspicion is not high.

Hysterectomy is the most common treatment option however; several types of conservative treatment have been used such as dilation curettage and excision of trophoblastic tissues using laparotomy or laparoscopy. Also, local and/or systemic MTX administration, bilateral hypogastric artery ligation, associated with dilation and evacuation under laparoscopic guidance and selective uterine artery embolization in combination with curettage and/or MTX injections. ${ }^{1}$

In our case, because of the hemodynamic status of our patient the only possibility was to carry out a laparotomy. The uterus was ruptured at the site of the upper end of the cervix and it was deemed unsafe to repair the uterus, consequently a subtotal hysterectomy was performed. In this decision contributed the fact that the patient was already contemplating an abortion, since she did not wish to have more children primarily because she was treated with etanercept for her rheumatoid arthritis. Etanercept although is not contraindicated in pregnancy it still has a questionable safety profile. ${ }^{6}$

\section{Acknowledgments}

None.

\section{Conflicts of interest}

The authors declare there is no conflict of interests.

\section{References}

1. Anisodowleh Nankali, Mina Ataee, Haleh Shahlazadeh, et al. Surgical management of the cesarean scar ectopic pregnancy: a case report. Case Reports in Obstetrics and Gynecology. 2013;2013:525187.

2. Srinivas M, Ragupathy K, Ndumbe F. Dilemma in the management of caesarean scar pregnancy. Reprod Syst Sex Disord. 2014;3:125.

3. Aniela Angerame Yela, Nathalia Marchiani. Conservative management of ectopic pregnancy in cesarean scar: case report. Rev Bras Ginecol Obstet. 2013;35(5):233-237.

4. Kamal Singh, Anjali Soni, Shelly Rana. Ruptured ectopic pregnancy in caesarean section scar: a case report. Case Reports in Obstetrics and Gynecology. 2012;2012:106892.

5. El-sayed El-badawy Awad, Ahmed Samy El-agwanya, Ahmed Mahmoud El-habashya, et al. Lower uterine segment pregnancy (Cesarean Scar Pregnancy and early placenta accreta): A rising complication from cesarean section with possible and similar early ultrasound diagnoses and management. The Egyptian Society of Radiology and Nuclear Medicine. 2015;46(4):977-980.

6. Clowse ME. The use of anti-TNF $\alpha$ medications for rheumatologic disease in pregnancy. Int $J$ Womens Health. 2010;2:199-209. 